\title{
IL Canone Linguistico Boccacciano, Non Senza Dissenso
}

\author{
Cecilia Casini*
}

ABSTRACT: Autore del massimo capolavoro in prosa volgare della letteratura medievale, Giovanni Boccaccio è stato determinante per la definizione del canone linguistico italiano, soprattutto a partire dalla codificazione cinquecentesca di Pietro Bembo. Ma non senza dissensi, se già all'indomani della pubblicazione delle Prose della volgar lingua sorgono le prime voci contrastanti, tese a favorire il modello di prosa offerto da Machiavelli.

PAROLE CHIAVE: Boccaccio; Questione della Lingua; canone; Machiavelli

RESUMO: Autor da maior obra-prima em prosa da literatura medieval em língua vulgar, Giovanni Boccaccio foi determinante para a definição do cânone linguístico italiano, sobretudo a partir da codificação proposta por Pietro Bembo no século XVI. Mas não sem divergências, uma vez que, logo após Bembo publicar seu Prose della volgar lingua, surgem as primeiras teses contrastantes que apoiam o modelo linguístico apresentado por Machiavelli.

PALAVRAS-CHAVE: Boccaccio; "Questão da Língua”; cânone; Machiavelli.

ABSTRACT: Author of prose's greatest masterpiece of medieval literature in the vernacular, Giovanni Boccaccio was crucial to defining the Italian language

\footnotetext{
* Universidade de São Paulo
} 
canon, especially since Pietro Bembo proposed its coding in the sixteenth century. Not without controversy, however, since shortly after the publication of Prose Della Volgar Language, Bembo presents the first contrasting theories that support the linguistic model presented by Machiavelli.

KEYWORDS: Boccaccio; "The Question of the vernacular Language”; canon; Machiavelli. 
_utore del massimo capolavoro in prosa volgare della letteratura medievale, Giovanni Boccaccio è stato determinante per la definizione del canone linguistico italiano, soprattutto a partire dalla codificazione cinquecentesca di Pietro Bembo. Il valore esemplare della lingua decameroniana sancisce definitivamente l'inclusione dell'autore nella triade delle Tre Corone, a incoronare, appunto, i tre più grandi esponenti della letteratura italiana (Dante, Petrarca, Boccaccio). La straordinaria diffusione del Decameron in Italia e in Europa e la sua fortuna presso la classe mercantile in ascesa, da un lato; la grandissima capacità della lingua di adattarsi agli usi più diversi, legati a situazioni e contenuti svariati, e il dominio perfetto della tecnica narrativa da parte dell'autore, dall'altro, contribuirono alla consacrazione di Boccaccio a modello per la prosa italiana, almeno fino alla riforma manzoniana di metà Ottocento. 
È lo stesso Boccaccio che dichiara la sua scelta di usare la lingua volgare in vari punti della sua opera, e non soltanto del Decameron. Si consideri per esempio il Filoloco, romanzo in prosa in cinque libri, scritto fra il 1336 e il 1339 a Napoli su richiesta di Fiammetta, la donna amata da Boccaccio. Nel prologo, Fiammetta si rivolge all' autore chiedendogli di comporre "un picciolo libretto volgarmente parlando" (BOCCACCIO, Filoloco; in QUAGLIO, 1967, p. 6); nell'epilogo Boccaccio si rivolge direttamente alla sua opera, secondo le modalità della letteratura classica, riaffermando l'impegno di scrivere in volgare e toccando altri temi che verranno poi ripresi e sviluppati, con maggiore ampiezza di respiro, nel Decameron (in particolare nel Proemio, nell'Introduzione alla IV giornata e nella Conclusione):

O piccolo mio libretto [...] dimora con lei [Fiammetta], ove io dimorare non oso, né di maggior fama avere sollecitudine, ché, con ciò sia cosa che tu da umile giovane sii creato, il cercare gli alti luoghi ti si disdice: e però agli eccellenti ingegni e alle robuste menti lascia i gran versi di Virgilio. A te la bella donna si conviene con pietosa voce dilettare, e confermarla ad essere d'un solo amante contenta. E quelli del valoroso Lucano, ne' quali le fiere arme di Marte si cantano, lasciali agli armigeri cavalieri insieme con quelli del tolosano Stazio. E chi con molta efficacia ama, il sermontino Ovidio seguiti, delle cui opere tu se' confortatore. Né ti sia cura di volere essere dove i misurati versi del fiorentino Dante si cantino, il quale tu sì come piccolo servidore molto dei reverente seguire. Lascia a costoro il debito onore, il quale volere usurpare con vergogna t'acquisterebbe danno. Elle son tutte cose da lasciare agli alti ingegni. La cicogna figliante nell'alte torri discende a vivere a' fiumi. A te bisogna di volare abasso, però che la bassezza t'è mezzana via. E Alcione volando batte le sue ali nelle salate onde, e vive. A te è assai solamente piacere alla tua donna, a cui è licito darti alto e basso luogo secondo che le piace: dalla quale, per mio consiglio, mai non ti partirai. E ove staresti tu meglio che nel suo grembo? (BOCCACCIO, Filoloco; in QUAGLIO, 1967, p. 652-653)

Dunque, la scelta di scrivere in volgare appare coerentemente integrata in un generale progetto di abbassamento di tono che va verso la definizione di una letteratura rivolta principalmente a un pubblico di estrazione borghese, quella nuova classe mercantile che con il suo dinamismo e spregiudicatezza aveva segnato l'originalità e la ricchezza della civiltà municipale italiana. 
Oltre a scegliere come destinataria dell'opera una donna (e, evidentemente, tutte le donne con lei) e a rivolgersi al libro ribadendone la "piccolezza" ("libretto"), l'autore lo invita a mostrarsi partecipe alle pene d'amore, dilettando Fiammetta con il racconto delle vicissitudini di Florio e Biancofiore, e a chiedere ispirazione alla poesia amorosa di Ovidio più che ai canti di guerra di Virgilio, Stazio e Lucano. Ancora, l'universo semantico del testo rimanda continuamente a valori ‘bassi' ("non oso", "umile”, "pietosa”, “contenta”, “confortatore”, "piccolo servidore”, "reverente seguire", "vergogna", "volare abasso", "mezzana via", "basso"), a sottolineare la rinuncia ad occuparsi di contenuti alti, per i quali il libro non avrebbe sufficiente competenza (il verbo "lasciare" appare declinato tre volte), in un interessante intreccio fra lessico di matrice cristiana e motivi cortesi che recupera la lezione di Dante (fra l'altro citato, e sempre presente in tutta l'opera di Boccaccio).

Come già sottolineato da tanta parte della critica boccacciana, l'esplicita dichiarazione dell'autore a favore di una letteratura così caratterizzata è una scelta a favore del realismo, pur con tutti i contenuti e le ambientazioni favolose di molta parte del Decameron, e culmina appunto nella composizione delle cento novelle narrate dai dieci giovani fiorentini riuniti in villa per sfuggire alla peste. Novelle che sono scritte, di fatto, nella lingua usata a Firenze alla metà del Trecento, quando i grandi rivolgimenti politici ed economici dei primi decenni del secolo avevano determinato notevoli spostamenti demografici all'interno della Toscana (soprattutto dalle città periferiche e dal contado verso Firenze), e provocato il sorgere di molti nuovi fenomeni linguistici che avevano mutato il fiorentino. Non intendiamo qui farne la lista, a fronte della ricca e documentatissima bibliografia esistente; quel che ci interessa è constatare l'accoglienza di questa lingua nella sua opera da parte di Boccaccio, e indicare come l'elaborazione stilistica e narrativa dell'autore si sia poi imposta fino al punto di farsi canone per la prosa italiana. Un canone esemplatosi su una lingua che però, nonostante la polimorfia dello stile, l'intrecciarsi dei vari registri linguistici, la forte connotazione di oralità dovuta alla mimesi del parlato e l'adozione spregiudicata di forme anche basse e triviali; nonostante, insomma, la succitata scelta realistica, rimane una lingua di natura prevalentemente aristocratica, la quale può parlare di qualsiasi cosa "mantenendo un superiore distacco", "compiacendosi della propria capacità di dire, della propria eleganza, del proprio decoro" (FERRONI, 2006, p. 180).

Questo carattere "nobile", di tono alto, che in gran parte ne giustificò l'elevazione a modello da parte dei grammatici cinquecenteschi, è stato messo in luce da numerosi studiosi. Vedasi per esempio come ne parla Auerbach, nell'analizzare la novella di Madonna Lisetta: 
Tanto la comare, che, con ipocrita cortesia e trattenendo il riso, esprime alcuni dubbi per meglio fare che la Lisetta parli, quanto costei che, per vanagloria, si lascia condurre perfino al di là della sua innata stupidaggine, parlano con perfetta verità e naturalezza. Tuttavia gli strumenti stilistici che il Boccaccio adopera non sono affatto popolari; la sua prosa tanto spesso analizzata, formata su antichi modelli e sui precetti retorici medievali, lascia giuocare tutti i suoi artifici: il riassunto di molti fatti nel periodo, il mutamento e l'incrocio dell'ordine delle parole al fine d'ottenere un rilievo delle cose più importanti, una cadenza più celere o più lenta, un'efficacia ritmico-melodica. [...] Tutto questo riesce già efficace mediante la brevità delle frasi che si incalzano, accavallandosi rapidissime e drammatiche; e proprio per questo, nonostante l'origine antica, dotta, delle forme stilistiche impiegate, non produce l'effetto di lingua scritta, ma rimane nel tono narrativo, tanto più che la posizione dei verbi, e con essa la lunghezza e il ritmo delle frasi più tranquille tra essi inserite, mutano di continuo in maniera piena d'arte e di spontaneità [...] Di tali artifici non se n'incontrano di certo nell'arte narrativa anteriore. (AUERBACH, 1979, p. 224-227)

Ai fini della ricostruzione dell'epoca in cui furono scritte le novelle, il lessico decameroniano è sicuramente l'elemento più rappresentativo, e anche quello che maggiormente contribuì al loro successo, come ci dimostra Paola Manni nel suo esaurientissimo testo del 2003, che abbiamo seguito in questa nostra ricostruzione e che citiamo in bibliografia. Secondo Manni (ibid.), esistono in particolare alcune tipologie di termini, afferenti svariati ambiti semantici, che ci permettono di apprezzare al meglio le implicazioni di carattere culturale di quella civiltà borghese di cui furono espressione. Seguiamo dunque l'accurata analisi della Manni e addentriamoci nel folto del testo boccacciano.

Assolutamente preponderante è la terminologia che riguarda l'ambiente mercantile (capi di abbigliamento, stoffe, oggetti, arredi casalinghi ecc.) e quella relativa all'ambito bancario, con tutta la serie delle espressioni tecniche connesse. Parole come i nomi delle monete sono esemplificativi di tutta quella fittissima rete di interconnessioni, economiche e culturali, che stava alla base della società medievale italiana. Numerosi sono anche i termini direttamente attinti dall' area giuridica (usati anche con intenti parodistici); i tecnicismi medici e della farmacopea; parole e espressioni attinenti all'ambiente marino (si considerino quante novelle si svolgono 
per mare!), che designano i vari tipi di imbarcazioni, le parti della nave e le diverse operazioni tecniche che vi si svolgevano. Termini vari di tipo tecnico vengono a volte usati per connotare allusivamente o ironicamente personaggi e situazioni, in modo da aumentarne l'espressività e la comicità. Per quanto riguarda il lessico di ascendenza colta, molto copiosa è la presenza di termini provenienti dalla tradizione lirica precedente e dalla poesia di Dante, grazie anche a citazioni, riecheggiamenti, ricorrenze ritmiche. Pertengono a questo tipo di lessico anche i gallicismi, soprattutto nelle novelle di ambientazione cavalleresca (come quella di Federigo degli Alberighi, tutta costruita sul tema della cortesia), e i latinismi, presenti soprattutto nelle parti più solenni e di maggiore elaborazione stilistica.

Accanto a scelte lessicali così impegnative, che ci confermano la natura sostanzialmente aristocratica della prosa boccacciana, è presente nel Decameron una grandissima varietà di forme linguistiche di tipo comune, molte delle quali passate poi all'italiano moderno (anche in accezioni diverse per la lingua della prosa e quella della poesia, come è il caso della coppia niuno/prosa - nessuno/poesia). Questo tipo di lessico, soprattutto quanto più popolarmente connotato, trova largo spazio nei dialoghi di stile più colloquiale e diretto, conferendo al ritmo linguistico naturalezza, grazia, vivacità. Di fatto, la caratteristica dell'oralità, tanto sottolineata dagli studiosi della lingua di Boccaccio, si dispiega in tutta la sua forza soprattutto nei dialoghi, in cui appaiono, spesso legati a situazioni comunicative di forte impatto emotivo, echi di lingua viva, di tipo popolare e eventualmente anche gergale, espressioni figurate, metafore. Vastissimo in particolare è il campo del lessico legato alla sessualità, soprattutto di tipo metaforico, che in molto contribuisce ad aumentare la varietà della lingua e delle situazioni dell'opera, confluendo poi nella terminologia erotica rinascimentale; e quello legato all'ingiuria (insulti, improperi, maledizioni, invettive), non di rado agito da personaggi femminili. Anche i modi di dire, le locuzioni, i proverbi legati all' ambito semantico dell'eros e dell'ingiuria, spesso alla loro prima apparizione nella letteratura in volgare, alimentano una fraseologia ricchissima, che conferiscono ai dialoghi la vivacità e la naturalezza tipiche del parlato, riflettendo "un impiego mediato [della lingua], niente affatto popolare, analogo a quello delle sentenze di provenienza colta, pure largamente presenti nel Decameron" (CHIECCHI, 1975-1976 apud MANNI, 2003, p. 295). Tanto le sentenze di origine dotta quanto i proverbi di matrice popolare si trovano soprattutto negli esordi e nelle conclusioni delle novelle, che sono i luoghi canonici dell'enunciazione delle leggi e della morale dimostrativa. Concludendo la lunga carrellata di esempi inerenti all'uso del lessico nelle novelle, la Manni sottolinea come la straordinaria ricchezza dei nomi propri, di 
persona e di luogo, sia in netto contrasto con l'esiguità e la genericità dei riferimenti onomastici della narrativa anteriore (MANNI, 2003, p. 296). Oltre a dare concretezza al relato, la varietà e ricchezza delle denominazioni ci permette di apprezzare il valore espressivo, funzionale alla narrazione, che l'autore consapevolmente attribuisce loro (per non parlare dell'inestimabile importanza di questo materiale per la ricostruzione della toponomastica dell'epoca): moltissimi sono i nomi che dichiarano significativamente il rapporto esistente con le caratteristiche fisiche e psicologiche dei personaggi, e che ancora oggi mantengono intatta la loro carica espressionistica (Guccio Imbratta, Buttafuoco, Scannadio, Erminio Avarizia, Chichibio ecc.), fino a suggerire valori antifrastici e caricaturali e a innescare sottili giochi di corrispondenze con altri nomi, interni o esterni alla novella. Boccaccio esplora con eccelsa abilità le potenzialità allusive contenute nei nomi sul piano del significante, mettendo in atto procedimenti scompositivi e anagrammatici che possono arrivare fino al non sense.

Ma ancor più del lessico, è la sintassi il tratto della prosa boccacciana destinato a esercitare l'influenza più forte sul successivo sviluppo della prosa italiana. E, di fatto, l'elaborazione di una sintassi in volgare che si ponesse sullo stesso piano di quello dei modelli classici latini, in modo da esserne nobilitata nella forma e nelle movenze, fu determinante nel processo di canonizzazione linguistica della lingua di Boccaccio da parte degli umanisti settentrionali. Ma allo stesso tempo tale elaborazione sintattica elevò moltissimo il grado di letterarietà linguistica del canone italiano, determinando di fatto l'inutilizzabilità della lingua per usi più precipuamente comunicativi o espressamente orali. Per inciso, vorremmo qui anticipare che fu soprattutto questo aspetto della lingua boccacciana che determinò allo stesso tempo buona parte delle reazioni negative nei suoi confronti - che pure vi furono, e anche prima della consacrazione bembiana -, e a provocare l'insofferenza generalizzata degli altri scrittori italiani verso il fiorentino trecentesco. La complessità della sintassi boccacciana e la sua alta patina di letterarietà vennero da taluni sentiti come ostacoli allo sviluppo di una lingua che fosse anche funzionale a un uso comunicativo; in tal senso, accenneremo più avanti a un unico caso, in cui a Boccaccio viene contrapposto l'esempio della lingua di Machiavelli. Tema che, vista l'ampiezza e la complessità, ci proponiamo di trattare più largamente in altra occasione.

Tornando al Decameron, nell'Introduzione alla IV giornata Boccaccio rivendica di aver scritto le sue "novellette" in volgare fiorentino "in istilo umilissimo e rimesso, quanto il più si possono"2 (1932, p. 243). Tale affermazione viene apparentemente smentita dalla complessità della sintassi che l'autore elabora per il testo, e che, meravigliosamente adattandosi a tutte le

2 Il referente immediato è l'Epistola a Cangrande, in cui Dante aveva parlato di "remissus [...] modus [loquendi] et humilis". 
diverse situazioni narrative dell'opera, la attraversa a tutti i livelli della diegesi ${ }^{3}$, creando un fitto intreccio di richiami e rimandi. È possibile individuare due principali nuclei di fenomeni sui quali si appunta in particolare l'intervento sintattico dell'autore: costrutti tipici dell'uso coevo e fenomeni riferibili a livelli espressivi di tipo corrente oppure a condizionamenti di natura dotta. Come sopra per il lessico, non è nostra intenzione descrivere qui dettagliatamente tale intervento, ma offrire un quadro generale di presentazione, sempre sulla scorta dell' analisi di Paola Manni ${ }^{4}$. Indicheremo pertanto i casi che, sulla base del modello boccacciano, meglio possono contribuire a delucidare le linee di sviluppo della tendenza arcaizzante della lingua letteraria italiana, così come si svolsero a partire dalla codificazione bembesca.

Fra i costrutti tipici dell'epoca di composizione del Decameron, risaltano in primo luogo quelli che riguardano la posizione dei pronomi atoni. A dimostrare la tendenza della prosa di Boccaccio a mantenersi legata alla tradizione, rimanendo al tempo stesso aperta verso le novità del tempo o addirittura precorrendole, il testo alterna il costrutto più antico, col tradizionale ordine accusativo-dativo, alla forma più recente, e già introdotta nel toscano d'inizio Trecento, del costrutto dativo-accusativo ${ }^{5}$. In certi casi i due costrutti possono apparire a brevissima distanza l'uno dall'altro: "Per me così vi vo' dire, donne mie care, che chi te la fa, fagliele; e se tu non puoi, tienloti a mente fin che tu possa" (V 10 64; in MANNI, 2003, p. 300). La legge Tobler-Mussafia, cioè quel fenomeno di area romanza secondo il quale la frase non può iniziare con un pronome atono, dovendosi quindi le particelle enclitiche unire al verbo cui si riferiscono, è sempre rispettata. L'enclisi e la proclisi sono variamente presenti. È assai presente anche il costrutto giustappositivo con la parola casa, relitto d'una tendenza all'uso del genitivo con i nomi propri tipico dell'italiano delle origini (“a casa Madonna Lisetta”, IV 2 15; in MANNI, 2003 , p. 301), con estensione a nomi comuni e in unione a un pronome.

Per quanto riguarda il verbo, è molto frequente l'uso con valore assoluto del trapassato remoto nella proposizione principale; così come il condizionale presente in dipendenza di un tempo storico per indicare l'idea del futuro al passato ("incontamente s'avisò che egli avrebbe novelle della donna" III 3 33; in MANNI, 2003, p. 302), secondo una prassi che arriverà fino a oltre Manzoni; mentre il condizionale passato, che oggi espleterebbe questa funzione, viene usato per indicare azioni rimaste virtuali, come quelle espresse nel periodo ipotetico dell'irrealtà.

\footnotetext{
3 Secondo Manni (2003, p. 315-316), si possono riconoscere quattro diversi livelli diegetici del testo decameroniano: quello più esterno e liminare, corrispondente alle "cornici" dell'opera, delle giornate e delle singole novelle (livello extradiegetico); un livello più interno, che riguarda più da vicino il messaggio narrativo in forma orale svolto dai dieci narratori, e che corrisponde all'Introduzione alla prima giornata, alle Introduzioni e alle Conclusioni alle singole novelle, ai testi di passaggio fra una novella e l'altra (livello intradiegetico); il livello diegetico propriamente detto, cositutito dalle novelle; il livello metadiegetico, corrispondente ai casi di racconto nel racconto.

4 Soprattutto alle pagine 298-325. Il testo della Manni contiene anche una ricchissima bibliografia specifica.

$5 \mathrm{Si}$ ricordi la difesa del Bembo dell'ordine accusativo + dativo per avvalorare la scelta classicistica, ancorché in parte frutto d'equivoco (Bembo attribuisce il costrutto al fiorentino moderno, mentre esso è già in crisi nello stesso Decameron).
} 
Molto vitale ancora la paraipotassi e la ripresa con sì della subordinata rispetto alla reggente.

Per quanto riguarda i fenomeni riferibili a livelli espressivi della lingua di tipo corrente, quello più vistoso è senz'altro la dislocazione, ancor oggi assai presente nell'italiano, soprattutto in quello parlato, anche se ufficialmente emarginata dalla norma. Quando si ha la dislocazione, il periodo boccacciano risulta quasi sempre sbilanciato a sinistra, grazie all'anticipazione di un elemento tematico, poi ripreso attraverso un pronome atono ("al re di Francia, per una nascenza che avuta avea nel petto e era male stata curata, gli era rimasta una fistola" III 97 , in MANNI, 2003, p. 303). Tale costrutto, molto usato in tutto il Decameron, può arrivare a notevoli gradi di complicazione (per esempio, con l'oggetto anticipato espresso da quello o da quello + sostantivo cui segue una relativa interposta, o costituito da un'intera proposizione). Anche se meno comunemente, appare anche la dislocazione a destra, cioè la posticipazione di un elemento tematico ripreso dal clitico in posizione cataforica ("Ma sallo Iddio che io malvolentier gli prendo" VIII 10 36; in MANNI, 2003, p. 304). In ogni caso, l'abbondanza dell'uso delle dislocazioni, sia nei dialoghi, sia nella narrazione, per caratterizzare espressivamente il testo sostenendolo con una sintassi complessa, pose il costrutto all'attenzione dei grammatici cinquecenteschi, a cominciare dallo stesso Bembo, e fu tema di accalorate discussioni.

Assai diffuso anche il fenomeno dell'anacoluto, soprattutto nella forma del "tema sospeso" (la frattura della congruenza sintattica che lega l'elemento tematico anteposto al pronome atono seguente), come nel seguente caso, definito da Momigliano "il più bell'anacoluto della nostra letteratura" (1924 apud MANI, 2003, p. 304): "Calandrino, se la prima gli era paruta amara, questa gli parve amarissima" (VIII 6 48; in MANNI, loc. cit.). Anche l'anacoluto, come le dislocazioni, può essere sintatticamente complicato fino a confondersi con un vero e proprio cambio di progetto da parte del narratore, anche per il suo prestarsi ad assumere particolari valenze espressive e stilistiche.

Pure molto presente è l'uso del che relativo indeclinato senza preposizione e senza marca di caso, che quasi si confonde col che polivalente anch'esso assai ricorrente nel testo. Ed è presente anche l'accordo ad sensum tra un nome collettivo singolare e un verbo al plurale, con vari livelli di complicazione del costrutto.

Per quanto riguarda l'organizzazione del periodo, si possono riscontrare varie particolarità, oggi sentite come agrammaticali, ma che paiono suggerite da una sintassi spontanea e colloquiale, probabilmente usata di fatto all'epoca in cui scrisse Boccaccio, e che serviva a garantire coesione testuale in un testo continuamente interrotto nel suo ordine lineare. Fra

6 Ci permettiamo di dissentire: per noi, il più bell'anacoluto della letteratura italiana è quello contenuto nella lettera di Niccolò Machiavelli a Francesco Vettori del 10 dicembre 1513, annunciante la composizione del Principe: "mi pasco di quel cibo che solum è mio e ch'io nacqui per lui". 
queste, la reduplicazione del che dopo l'interposizione di una parentetica, anch'essa variamente complicata; e la coordinazione di un gerundio o di un participio passato con un verbo di modo finito, o di gerundio e di indicativo ("Ora avvenne che, essendo il tempo caldo e molte brigate di donne e di cavalieri [...] andassero a diportarsi a' liti del mare [...], Ricciardo [...] similmente con sua compagnia v'andò" III 6 9, in MANNI, 2003, p. 307; "E essendo ogni cosa presta (e niun'altra cosa che la venuta del marchese era da lei aspettata) avvenne che un fante giunse alla porta" II 2 20, in MANNI, loc. cit.).

I fenomeni riferibili ai condizionamenti di natura dotta sono quelli che maggiormente caratterizzano la prosa del Decameron, quelli in cui la maestria dell'elaborazione raggiunge il suo apice e che, come si è visto, più si imporranno all'attenzione e all'ammirazione dei grammatici cinquecenteschi. Fra tali fenomeni stanno in primo luogo le inversioni. La casistica è molto vasta: dalle microsequenze che antepongono il participio all'ausiliare (il già citato "la venuta del marchese era da lei aspettata", II 2 20; in MANNI, loc. cit.), o che lo inseriscono fra il verbo servile e l'infinito ("lasciata oltre la giovane andare" II 5 5; in MANNI, 2003, p. 309), fino ai casi in cui vengono coinvolti i costituendi fondamentali della frase, sovvertendone l'andamento (" a' suoi fratelli ciò che veduto aveva la passata notte d'Elisabetta e di Lorenzo raccontò" IV 5 7, in MANNI, loc. cit.; "Aveva alle sue parole già Filomena fatta fine” X 92 , in MANNI, loc. cit.). La pratica dell'inversione, audacemente realizzata da Boccaccio sul modello della prosa latina, lo porta a infrangere l'ordine lineare attraverso una studiata collocazione delle parole, attenta anche agli effetti ritmico-melodici della frase e al ritmo del cursus medievale. La sintassi, dominata dall'ipotassi, risulta enormemente dilatata e tortuosa, con accumulo di subordinate gerarchicamente organizzate, ulteriormente complicate da incisi parentetici, con la reggente relegata alla fine del periodo. I due brani seguenti sono un esempio dell'estrema complicazione a cui può giungere il periodo:

Il quale un giorno dietro mangiare là giù venutone, essendo la donna, la quale Ghismonda aveva nome, in un suo giardino con tutte le sue damigelle, in quella senza essere stato da alcuno veduto o sentito entratosene, non volendo lei torre dal suo diletto, trovando le finestre della camera chiuse e le cortine del letto abbattute, a piè di quello in un canto sopra un carello si pose a sedere. (IV 1 17; in MANNI, 2003, p. 309) 
Ma Guccio Imbratta, il quale era più vago di stare in cucina che sopra i verdi rami l'usignuolo, e massimamente se fante vi sentiva niuna, avendone in quella dell'oste una veduta, grassa e grossa e piccola e mal fatta, con un paio di poppe che parean due ceston de letame e con un viso che parea de' Baronci, tutta sudata, unta e affumicata, non altramenti che si gitti l'avvoltoio alla carogna, lasciata la camera di frate Cipolla aperta e tutte le sue cose in abbandono, là si calò. (VI 10 21; in MANNI, 2003, p. 309-310)

I due brani ci permettono fra l'altro di notare come un espediente di così nobile origine (la prosa classica in latino) venga indifferentemente usato da Boccaccio per due situazioni narrative molto diverse: la prima, di carattere aulico e elevato; la seconda, di tipo decisamente plebeo a popolare.

Lo schema sintattico del latino è ricalcato anche nel caso delle numerosissime oggettive che presentano l'accusativo + l'infinito (il già citato "non volendo lei torre dal suo diletto", IV 1 17; in MANNI, 2003, p. 309), come nella lunghissima sequenza di cinque oggettive del brano seguente:

Confessò Bernabò così essere fatta la camera come diceva e oltre a ciò sé riconoscere quelle cose veramente della sua donna essere state; ma disse lui aver potuto da alcuno de' fanti della casa sapere le qualità della camera e in simil maniera avere avute le cose; per che, se altro non dicea, non gli parea che questo bastasse a dovere aver vinto. (II 931 ; in MANNI, 2003, p. 310)

Un' altra costruzione di chiara origine latina è quella dei verbi temere e dubitare + negazione ("temo che infamia e riprensione, senza nostra colpa o di loro, non ce ne segua se gli meniamo" I Intr. 83; in MANNI, loc. cit.); "Li due fratelli, li quali dubitavan forte non ser Ciappelletto gl'ingannasse" I 1 78; in MANNI, loc. cit.).

Anche il gerundio assoluto, semplice o composto, tanto usato da Boccaccio nelle novelle per la grande versatilità semantica nell'esprimere rapporti subordinanti di tipo diverso (principalmente temporali e causali), è un calco diretto dell'ablativo assoluto latino: "E partendosi Tingoccio da lui, Meuccio si ricordò della comare" (VII 10 24; in MANNI, 2003, p. 311). Minore ma pur sempre notevole la presenza del participio presente assoluto: "Il quale, sì come Savio, mai, vivente il re, non la scoperse" (III 2 32; in MANNI, loc. cit.). Sempre riguardo il gerundio e il participio presente, essi possono essere usati con valore attributivo, secondo la prassi dei volgarizzamenti e della prosa letteraria del Due-Trecento: “e [il lupo] lei gridante aiuto si 
sforzava di tirar via" (IX 7 6; in MANNI, loc. cit.).

Molto comune anche l'uso del relativo all'inizio del periodo, in modo tale da proporre, a partire dal recupero del valore coordinante e dimostrativo della coniunctio relativa, già rafforzatosi nel latino tardo e medievale, un'alternativa "colta" della funzione dei pronomi relativi per esprimere legami di natura paratattica (in pratica, in questi casi Boccaccio usa i relativi come puri connettivi).

Come si è visto, è grandissima la varietà stilistica presente nell'opera boccacciana, tanto sul piano dei contenuti (le differenti situazioni delle novelle), quanto su quello dei diversi piani narrativi. E di fatto la variatio, e l'eccelsa perizia dell'autore nell'orchestrarla, fu uno degli elementi che richiamarono favorevolmente l'attenzione dei grammatici del Cinquecento sul Decameron; per il Salviati, per esempio, "in quel libro sono in sovrana eccellenza in vari luoghi sparsi tutti gli stili e per lui solo possiam dir quase d'avere e pregiati autori e pregiate scritture di tutte le maniere" (SALVIATI apud MANNI, 2003, p. 316). Ma sopra tutti gli altri, furono gli aspetti arcaizzanti della prosa di Boccaccio a determinare la sua inclusione nel canone da parte di Bembo. Come si sa, la soluzione bembiana nell'ambito della Questione della lingua fu una proposta di tipo estetico-stilistico, a favore del recupero della pretesa perfezione originaria della lingua del secolo XIV contro la corrente fiorentinista dell'uso vivo e quella eclettico-cortigiana. È in tal senso che l'eleganza classica della prosa di Boccaccio e la sua cosciente "letterarietà" vengono indicate come modello di riferimento nelle Prose della volgar lingua del 1525.

Ma non senza dissensi, se già all'indomani della pubblicazione delle Prose Gabriele Cesano, per citare una fonte di prima mano, si sarebbe pronunciato contro Boccaccio, a favore di Machiavelli, in una conversazione letteraria alla corte estense di Ferrara. Ce ne dà testimonianza l'antitoscanista Girolamo Muzio nelle sue Battaglie di Hieronimo Mutio Giustinopolitano, pubblicate a Venezia nel 1582, schierandosi a difesa di Boccaccio, lui, Muzio, contro appunto il Cesano, che invece difende Machiavelli. Vale la pena riprodurre tutto il brano tratto dalle Battaglie, a riprova della foga polemica suscitata dal tema:

Io non credea, M. Gabriello \& M. Bartholomeo, che da alcuno si dovesse dubitare tra ló stilo del Boccaccio \& del Macchiavelli quale havesse ad esser reputato il più leggiadro. Anzi ho io sempre stimato che in questa nostra età ci siano di quegli che di purità di lingua, \& di dolcezza, \& di altezza di dire avanzino il Macchiavelli: ma che alcuno non ce ne habbia, che al Boccaccio meriti di essere agguagliato. Or voi 
(per quanto io comprendo) havete opinione a questa contraria: \& tanto la havete voi contraria, che dite il dir del Macchiavelli essere dell'altro senza comparation più bello $[\ldots]$ Et dico, che de' libri del Macchiavelli già è gran tempo che me ne vennero alcuni in mano: \& havendone nella lettion di poche righe il suo stilo $\&$ la sua lingua notata, gli gittai da parte, come quegli da' quali io non pensava di poter raccogliere cosa di tanta utilità, di quanto danno potrebbe essere stato quel suo dire alle mie scritture. Né da poi mi è mai venuta voluntà di tornargli a vedere, anzi, più me ne ha tenuto lontano l'havere io udito dire che ne' libri di lui niuna pietà, niuna humanità, niuna religione vi si trova, ma che sono tutti pieni di ammaestramenti di crudeltà, di tirannia \& di infidelità. Hora sentendogli da voi di tanto proporre nello stilo a quegli del Boccaccio, ho voluto fra prova se co'1 condimento del giudicio vostro io ne potessi sentire alcun dilettevole sapore, \& a leggerne alcune poche carte mi sono condutto [...]. Io non so trovar nelle parole di lui cosa che comportabile mi paia in iscrittore che voglia con lode alcuna cosa scrivere. Se riguardo alla forma del dire, non so come dir si possa più bassamente. Se cerco degli ornamenti, non ne trovo niuno; anzi mi pare egli esser tutto secco, \& digiuno di ogni leggiadria. Poi nella lingua egli è tale che, oltra l'usar molte parole latine Lá dove non men belle ne haverebbe havute delle volgari, \& nella variatione \& nella proprietà de' verbi egli è tutto cieco; usa male i nomi \& peggio i pronomi; non sa ben collocare né articoli, né adverbii: \& insomma, tanto sa delle osservationi della lingua, quanto chi non ne sa niente... (CASTELLANI POLIDORI, 1978, p. 82-83)

Boccaccio o Machiavelli, dunque? Sappiamo dalla storia che a imporsi fu il modello boccacciano, segnando fortemente di sé lo sviluppo futuro della prosa italiana e connotandone l'eccesso di letterarietà, almeno fino a quasi tutto l'Ottocento. Al di là di ogni giudizio finale, comunque, ci sembra felice l'epoca in cui il canone letterario italiano poteva oscillare fra questi autori. 


\section{Riferimenti bibliografici}

AUERBACH, E. Mimesis. Il realismo nella letteratura occidentale. Turim: Einaudi, 1979.

BOCCACCIO, G. Decameron. Milão: Hoepli, 1932.

CASTELLANI POLIDORI, O. Niccolò Machiavelli e il "Dialogo intorno alla nostra lingua". Florença: Olschki, 1978.

DURANTE, M. Dal latino all'italiano moderno. Saggio di storia linguistica e culturale. Bolonha: Zanichelli, 1981.

FERRONI, G. Profilo storico della letteratura italiana. v.I. Turim: Einaudi, 2006.

MANNI, P. Storia della lingua italiana. Il Trecento toscano. Bolonha: Il Mulino, 2003.

PATOTA, G. I percorsi grammaticali. In SERIANNI, L.; TRIFONE, P. (orgs.). Storia della lingua italiana. v.I. I luoghi della codificazione. Turim: Einaudi, 1993.

QUAGLIO, A. E. (org.). Giovanni Boccaccio, Filoloco, in Tutte le opere di Giovanni Boccaccio, v.I. Milão: Mondadori, 1967.

STOLZ, F.; DEBRUNNER, A.; SCHMID, W. P. Storia della lingua latina. Bologna: Pàtron, 1973.

STUSSI, A. Lingua. In BRAGANTINI, R.; FORNI, M. (orgs.). Lessico critico decameroniano. Turim: Bollati Boringhieri, 1995. 